\title{
Extracted atmospheric impairments on earth-sky signal quality in tropical regions at Ku-band
}

\begin{abstract}
Atmospheric condition variations were shown to have a major effect on the earth sky signal quality at $\mathrm{Ku}$ band. Moreover, such variations increased in the tropical regions as compared to temperate areas due to their different weather parameters. With the increase of recent satellite communication technology applications throughout the tropical countries and lack of information regarding the atmospheric impairments analysis, simulation and mitigation techniques, there is an ever increasing need for extracting a unique and accurate performance of the signal quality effects during highly natural tropical weather impairments. This paper presents a new method developed for proper analysis with distinctive and highly realistic performance evaluation for signal quality during the atmospheric conditions variations in 14 tropical areas from the four continents analyzed based on actual measured parameters. The method implementation includes signal attenuation, carrier to noise ratio, symbol energy to noise ratio, and symbol error rate at different areas and different modulation schemes. Furthermore, for improvement in analysis in terms of covering more remarkable regions in tropics, the paper provides new measurements data with analysis for certain region in tropics used as a test bed and to add measurement data of such area to the world's data base for future researchers. The results show a significant investigation and performance observation in terms of weather impairments in tropical regions in general and each region in that area in particular regarding the signal attenuation and error rates accompanied for several transmission schemes.
\end{abstract}

Keyword: Atmospheric impairments; Rain attenuation; Tropical regions; Ku-band satellite communication; Carrier to noise ratio; Symbol error rate 